\title{
Molecular characteristics of acid and pepsin soluble collagens from the scales of golden carp (Probarbus jullieni)
}

\author{
Ali Muhammed Moula Ali ${ }^{1}$, Soottawat Benjakul1,* and Hideki Kishimura ${ }^{2}$ \\ ${ }^{1}$ Department of Food Technology, Faculty of Agro-Industry, Prince of Songkla University, Hat Yai, Songkhla, Thailand, ${ }^{2}$ Laboratory of Marine \\ Products and Food Science, Research Faculty of Fisheries Sciences, Hokkaido University, Hakodate, Hokkaido, Japan
}

\section{A B S TR A C T}

\begin{abstract}
Acid soluble collagen (ASC) and pepsin soluble collagen (PSC) were isolated from the scales of golden carp (Probarbus jullieni). Both ASC and PSC, identified as type I collagen, contained $\alpha$ - and $\beta$-chains as the dominant constituents. ASC and PSC had the yields of 0.42 and $1.16 \mathrm{~g} 100 \mathrm{~g}^{-1}$ (dry weight basis), respectively. Amino acid composition revealed that glycine constituted $1 / 3$ of total amino acid residues and no cysteine was found. ASC and PSC had imino acid contents of 197 and 202 residues/1000 residues, respectively. Fourier transform infrared spectroscopy (FTIR) and circular dichroism (CD) spectra indicated high integrity of the triple helical structure of both collagens. Based on differential scanning calorimetry (DSC), ASC and PSC had $\mathrm{T}_{\max }$ of 37.67 and $37.83^{\circ} \mathrm{C}$, respectively. Both collagens exhibited

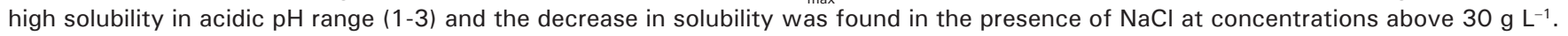
The overall results demonstrated that scales of golden carp could serve as another source of collagen.
\end{abstract}

Keywords: Acid soluble collagen; Golden carp; Fish scales; Pepsin soluble collagen

\section{INTRODUCTION}

Collagen is a primary component that provides mechanical support to extracellular matrix and helps to regulate cell from the surrounding (Kielty et al., 1993). Collagen is categorised as fibrous protein and constitutes up to $30 \%$ of total protein. There are over 28 types of collagen distributed in cartilage, tendons, ligaments, skins, bones, and other organs (Jongjareonrak et al., 2005). Collagen has been widely used in food, cosmetic, pharmaceutical and biomedical industries. This may be due to its weak antigenicity with excellent biocompatibility/ biodegradability in nature (Rizk and Mostafa, 2016). Since collagen from porcine or bovine sources has the limitation due to religious concern (Kittiphattanabawon et al., 2005), the demand for alternative commercial sources of collagen is increasing.

Fish processing generates a considerable amount of organic by-products up to $50-70 \%$, depending on fish species. To increase the value of those by-products, marketable value- added products, especially collagen and gelatin, have gained increasing interest for manufacturers. Collagens from bone, skin and scale of common carp (Cyprinus carpio) (Duan et al., 2009), skin of bigeye snapper (Benjakul et al., 2010), scale of snakehead (Ophiocephalus argus) (Liu et al., 2008) and swim bladder of seabass (Sinthusamran et al., 2013) have been extracted. Nevertheless, biochemical properties of collagen can be influenced by species, extraction procedures and other factors. Collagens from aquatic animals are gaining attention for various potential applications as they are free of disease transmission and have no dietary restrictions (Liu et al., 2012).

Golden carp (Probarbus jullieni) is a freshwater fish, commonly distributed in Southeast Asian river basins. Golden carp is very popular and primarily aquacultured in Thailand and Lao PDR. Based on global aquaculture production, the annual growth rate was $3.86 \%$, increasing from 3077 metric ton in 2005 to 5500 metric ton in 2010 (FAO 2016). During descaling process, scales are generated as a leftover (Zall, 2004). Commonly, scales have been

\footnotetext{
*Corresponding author:

Soottawat Benjakul, Department of Food Technology, Faculty of Agro-Industry, Prince of Songkla University, Hat Yai, Songkhla, 90112, Thailand. Tel: 66-7428-6334, Fax: 66-7455-8866, E-mail: soottawat.b@psu.ac.th
} 
considered as feed material with low market value or discarded as a waste, thus contributing to pollution. Those scales can serve for extraction of marketable biomolecules, especially collagen. Nevertheless, no information on collagen from scales of golden carp exists. The present study aimed to extract and molecularly characterise collagen from scales of golden carp.

\section{MATERIALS AND METHODS}

\section{Chemicals}

Acetic acid and sodium hydroxide were obtained from Merck (Darmstadt, Germany). Pepsin from porcine (EC3.4.23.1) (516 U/mg dry matter) and protein molecular weight markers were purchased from GE Healthcare UK (Aylesbury, UK). Coomassie Blue R-250, sodium dodecyl sulphate (SDS) and N,N,N',N'-tetramethylethylenediamine (TEMED) were purchased from Bio-Rad Laboratories (Hercules, CA, USA). All chemicals were of analytical grade.

\section{Preparation of golden carp scales}

The scales of golden carp (Probarbus jullieni) having an average body weight of 6-8 $\mathrm{kg}$ were procured from the fish dock located in Mahachai, Samut Sakhon Province, Thailand. Scales $(30 \mathrm{~kg}$ ) were packed in a polyethylene bags $(5 \mathrm{~kg} / \mathrm{bag})$, containing with ice using scale/ice ratio of $1: 3(\mathrm{w} / \mathrm{w})$. Transportation was carried out using insulated polystyrene box. Upon arrival, scales were washed thoroughly in cold water to remove the surface contaminants. Scales were then drained on a screen for $10 \mathrm{~min}$ and packed in zip lock polyethylene bags. The samples were kept $-20{ }^{\circ} \mathrm{C}$ for further use (not more than two months). When required, the scales were subjected to thawing under running tap water for about $20 \mathrm{~min}$. Scales of golden carp contained $16.24 \pm 0.18 \%$ moisture, $40.36 \pm 0.27 \%$ protein, $0.62 \pm 0.1 \%$ fat, and $42.16 \pm 0.21 \%$ ash as analysed using the AOAC method (AOAC, 2000). Hydroxyproline content of scale was $22.46 \pm 0.82 \mathrm{mg} \mathrm{g}^{-1}$ of sample, determined as detailed by Bergman and Loxley (1963).

\section{Extraction of collagen from scales of golden carp}

All the procedures for pretreatment, washing and extraction were carried out at $4{ }^{\circ} \mathrm{C}$ to avoid denaturation of collagen.

\section{Extraction of acid soluble collagen (ASC)}

Extraction was performed following the method of Chuaychan et al. (2015) with a slight modification. Before extraction, scales were treated with $0.1 \mathrm{M} \mathrm{NaOH}$ at the scale/alkaline solution ratio of 1:10 (w/v) for $8 \mathrm{~h}$ for the removal of non-collagen proteins. The process was carried out with continuous stirring with the aid of overhead stirrer (W20.n, IKA®-Werke GmbH \& CO.KG, Stanfen, Germany). Alkaline solution was freshly replaced for every $4 \mathrm{~h}$. Subsequently, scales were thoroughly washed using iced water until the wash water had the neutral $\mathrm{pH}$. These scales were further subjected to demineralisation using 0.5 M EDTA-2Na salt solution of $\mathrm{pH} 7.4$ at the ratio of 1:10 (w/v) under the stirring condition for about $48 \mathrm{~h}$. EDTA solution was freshly replaced after $24 \mathrm{~h}$. Finally, the demineralised scales were washed with iced water till the wash water had neutral $\mathrm{pH}$.

After demineralisation, ASC was extracted using $0.5 \mathrm{M}$ acetic acid with the scale/acid solution ratio of 1:15 (w/v) for $48 \mathrm{~h}$ under stirring condition. Thereafter, the mixture was filtered with double-layered cheesecloth. The filtrate was collected and collagen was precipitated by salting out. To the filtrate, $0.05 \mathrm{M}$ tris (hydroxymethyl) aminomethane (pH 7.0) was added, and $\mathrm{NaCl}$ was added slowly to obtain a concentration of $2.5 \mathrm{M}$. The pellet was recovered by centrifugation at $15,000 \times \mathrm{g}$ at $4{ }^{\circ} \mathrm{C}$ for $1 \mathrm{~h}$ using a centrifuge model CR 22GIII (Hitachi, Tokyo, Japan). The pellet was redissolved in the small volume of $0.5 \mathrm{M}$ acetic acid and then dialysed with 20 volumes of $0.1 \mathrm{M}$ acetic acid for about $48 \mathrm{~h}$. Thereafter, the solution was further dialysed in 20 volumes of double distilled water for $72 \mathrm{~h}$. Finally, collagen was freeze-dried using a freeze-dryer model CoolSafe 55 (ScanLaf A/S, Lynge, Denmark). The resulting collagen referred to as 'acid soluble collagen; ASC' was analysed.

\section{Extraction of pepsin soluble collagen (PSC)}

The undissolved residue gained after ASC extraction was used to extract PSC. The residue was extracted with acetic acid $(0.5 \mathrm{M})$ containing $1 \%$ pepsin $(\mathrm{w} / \mathrm{w})$ at a residue/solution ratio of $1: 10(\mathrm{w} / \mathrm{v})$. The extraction was performed with the aid of continuous stirring for $24 \mathrm{~h}$ at $4{ }^{\circ} \mathrm{C}$. Subsequently, the extracted collagen was filtered and precipitated in the similar fashion as described for ASC. Dialysis and lyophilisation were carried out as detailed above. The obtained collagen termed 'pepsin soluble collagen, PSC' was subjected to analysis.

\section{Analysis}

Both collagens, ASC and PSC, were calculated for yield and further analysed.

\section{Yield}

The yield was calculated based on the dry weight of scales.

Yield $(\%)=($ Weight of freeze-dried collagen $) /($ Weight of initial dry scales) $\times 100$

\section{Amino acid composition}

Amino acid composition was determined as described by Nagarajan et al. (2012) with some minor modifications. 
In brief, the samples were vacuum-hydrolysed in the presence of $4 \mathrm{M}$ methanesulphonic acid consisting of $0.2 \%(\mathrm{v} / \mathrm{v}) 3-2\left(2\right.$-aminoethyl) indole at $115^{\circ} \mathrm{C}$ for approximately $24 \mathrm{~h}$. Acid hydrolysed samples were then neutralised using $3.5 \mathrm{M} \mathrm{NaOH}$. Samples were further diluted using $0.2 \mathrm{M}$ citrate buffer ( $\mathrm{pH} 2.2$ ), and $0.04 \mathrm{~mL}$ of aliquot was loaded onto an amino acid analyser model MLC-703 (Atto Co., Tokyo, Japan). An automated liquid chromatography system was used in combination with ninhydrin post column derivatisation. The analysis was done in duplicate.

\section{Sodium dodecyl sulphate-polyacrylamide gel electrophoresis (SDS-PAGE)}

ASC and PSC were determined for protein patterns using SDS-PAGE as per the method of Laemmli (1970). Sample (12 $\mu \mathrm{g}$ protein), examined by the Biuret method (Gornall et al., 1949), was loaded into polyacrylamide gel with $4 \%$ stacking gel and $7.5 \%$ running gel. After proteins were separated, staining and destaining were performed as described by Chuaychan et al. (2015). The band intensity of proteins were quantified with the public domain digital analysis software called ImageJ 1.42q, (Bethesda, MD, USA).

\section{Fourier transform infrared spectroscopy (FTIR)}

FTIR spectra were analysed as described by Sinthusamran et al. (2013). The scans of samples were performed within the range of $650-4000 \mathrm{~cm}^{-1}$ using a resolution rate of $4 \mathrm{~cm}^{-1}$ for 32 scans against a background. Spectrum was recorded at $25{ }^{\circ} \mathrm{C}$ using FTIR spectrometer model Equinox 55 (Bruker, Ettlingen, Germany). Spectral data was analysed using the OPUS 3.0 data collection software programme (Bruker, Ettlingen, Germany).

\section{Circular dichroism (CD)}

ASC and PSC $\left(2.0 \times 10^{-4} \mathrm{gL}^{-1}\right)$ were prepared by dissolving in $0.5 \mathrm{M}$ acetic acid. The spectra were obtained via scanning in the wavelength range of 190 to $240 \mathrm{~nm}$ using JASCO J-801 spectrometer with a bandwidth rate of $1 \mathrm{~nm}$. The measurements were recorded at $25{ }^{\circ} \mathrm{C}$ by maintaining nitrogen gas as an atmosphere. Acetic acid $(0.5 \mathrm{M})$ was used as blank, which was then subtracted from the average of sample spectra. The obtained results were expressed as the molar residual ellipticity.

\section{Zeta potential}

ASC and PSC (0.02\%) in $0.05 \mathrm{M}$ acetic acid (w/v) were prepared. Approximately, $20 \mathrm{~mL}$ of solution was subjected to autotitrator model BI-ZTU (Brookhaven Instruments Co., Holtsville, NY, USA) over the range of $\mathrm{pH} 2$ to 12 using either $1.0 \mathrm{M}$ nitric acid or 1.0 M KOH. The zeta potential at different $\mathrm{pHs}$ was recorded by zeta potential analyser, model ZetaPALs (Brookhaven Instruments
Co., Holtsville, NY, USA). The isoelectric point (pI) was assessed from $\mathrm{pH}$ rendering the zeta-potential of zero.

\section{Differential scanning calorimetry (DSC)}

DSC was run as described by Kittiphattanabawon et al. (2005) with a slight modification. Both ASC and PSC were subjected to rehydration in deionised water using a solid/ solution ratio of $1: 40(\mathrm{w} / \mathrm{v})$ at $4{ }^{\circ} \mathrm{C}$ for $24 \mathrm{~h}$. The scan was performed over the temperature range of $20-50{ }^{\circ} \mathrm{C}$ with the rate of $1{ }^{\circ} \mathrm{C} \min ^{-1}$ using a differential scanning calorimeter (Perkin-Elmer Model DSC7, Norwalk, CA, USA). Endothermic peak representing the maximum transition temperature $\left(\mathrm{T}_{\max }\right)$ was examined. Total denaturation enthalpy $(\Delta \mathrm{H})$ was determined from the area of DSC thermogram.

\section{Solubility}

The relative solubility of ASC and PSC was estimated as per Jongjareonrak et al. (2005) with a minor modification. Collagens were dissolved in acetic acid $(0.5 \mathrm{M})$ to have a final concentration of $3 \mathrm{mg} \mathrm{mL}^{-1}$. Aliquots were adjusted to the designated $\mathrm{pH}$ values (1-10) using either $6 \mathrm{~N} \mathrm{NaOH}$ or $6 \mathrm{~N} \mathrm{HCl}$. The final volume was adjusted to $10 \mathrm{~mL}$ by distilled water with $\mathrm{pH}$ adjustment to the corresponding value.

$\mathrm{NaCl}$ at different final concentrations of $0,10,20,30,40$, 50 and $60 \mathrm{~g} \mathrm{~L}^{-1}$ was used to examine its effect on collagen solubility $\left(3 \mathrm{mg} \mathrm{mL}^{-1}\right)$. Firstly, the mixtures of collagen in salt solution were prepared. The mixtures were stirred continuously for $1 \mathrm{~h}$ at $4{ }^{\circ} \mathrm{C}$ and then centrifuged at $8500 \times \mathrm{g}$ for $20 \mathrm{~min}$. The resultant supernatant was used for examining the protein content by the Lowry method (Lowry et al., 1951). Bovine serum albumin was used as a protein standard.

\section{Statistical analysis}

All the experiments were conducted in triplicates, and a completely randomised design (CRD) was used. Analysis of variance (ANOVA) was performed and mean comparison was made by the Duncan's multiple range tests. The analysis was conducted using an SPSS statistical package (SPSS 11.0 for Windows, SPSS Inc, Chicago, IL, USA).

\section{RESULTS AND DISCUSSION}

\section{Yield of ASC and PSC}

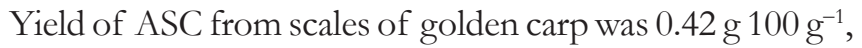
which was slightly higher than that reported for ASC from seabass scales (0.38 g $\left.100 \mathrm{~g}^{-1}\right)$ (Chuaychan et al., 2015) but was slightly lower than that of golden goatfish scales ASC,

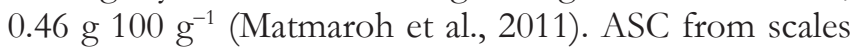
of rohu was reported to have relatively high yield, about

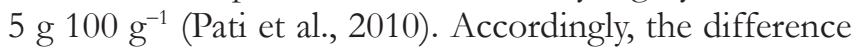


in yield of ASC depends on the type of fish, age and structure of collagen in scale matrix, etc (Duan et al., 2009).

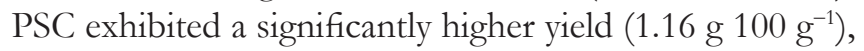
compared to ASC. The yield was increased up to 3.8 folds when pepsin was used as the extraction aid. As pepsin has been reported to cleave precisely at telopeptide region (Nalinanon et al., 2007), the cleaved collagen was released in a significant amount. The result showed that pepsin increased the extraction efficiency of collagen from the scales of golden carp.

\section{Amino acid compositions}

Amino acid compositions of ASC and PSC were expressed as residues/1000 amino acid residues as shown in Table 1. Glycine constituted nearly one-third of total amino acids (330-333 residues/1000 residues) for ASC and PSC. Glycine at every third position displays the main role in sinking the steric interference within the $\alpha$-chain. It is mainly involved in intermolecular hydrogen-bonding perpendicular to the helical axis of collagen (Fontaine-Vive et al., 2009). No cysteine was present in both collagens. This confirmed that there were no disulphide bonds in the collagen. The content of imino acids including proline and hydroxyproline in ASC (197 residues/1000 residues) was slightly lower than that of PSC (202 residues/1000 residues). The removal of telopeptide caused by pepsin more likely affected imino acids of collagen to some degree. The imino acid contents of collagen from golden carp scales were higher

\begin{tabular}{l}
$\begin{array}{l}\text { Table 1: Amino acid composition of acid soluble } \\
\text { collagen (ASC) and pepsin soluble collagen (PSC) from the } \\
\text { scales of golden carp }\end{array}$ \\
\begin{tabular}{lcc} 
Amino acids & $\begin{array}{c}\text { ASesidues/1000 } \\
\text { residues) }\end{array}$ & $\begin{array}{c}\text { PSC (Residues/1000 } \\
\text { residues) }\end{array}$ \\
\hline Alanine & 120 & 115 \\
Arginine & 52 & 49 \\
Aspartic acid/ & 48 & 45 \\
asparagine & & \\
Cysteine & 0 & 0 \\
Glutamine/ & 69 & 69 \\
glutamic acid & & \\
Glycine & 330 & 333 \\
Histidine & 4 & 6 \\
Isoleucine & 12 & 13 \\
Leucine & 23 & 23 \\
Lysine & 26 & 25 \\
Hydroxylysine & 7 & 8 \\
Methionine & 12 & 11 \\
Phenylalanine & 14 & 13 \\
Hydroxyproline & 80 & 86 \\
Proline & 118 & 116 \\
Serine & 36 & 36 \\
Threonine & 24 & 25 \\
Tyrosine & 4 & 4 \\
Valine & 22 & 22 \\
Imino acid & 197 & 202 \\
\hline & & \\
\hline & &
\end{tabular} \\
\hline
\end{tabular}

than those of seabass (193 and 195 residues/1000 residues) (Chuaychan et al., 2015) and collagen from scales of carp (192 residues/1000 residues) (Duan et al., 2009). In general, hydroxyproline and proline are strengthening units in triple helix through hydrogen bonds (Li et al., 2008) and also contribute to the thermal stability of collagens (Liu et al., 2012). Alanine (115-120 residues/1000 residues), arginine (49-52 residues/1000 residues), asparagine/aspartic acid (45-48 residues/1000 residues), and glutamine/glutamic acid (69 residues/1000 residues) were present at high amount. However, tyrosine (4 residues/1000 residues) as well as histidine (4-13 residues $/ 1000$ residues) were found at very low amount in both ASC and PSC. Lysine contents in ASC and PSC were 26 and 25 residues/1000 residues, respectively. Hydroxylysine was present at small amounts (7-8 residues/1000 residues). Lysine and hydroxylysine, particularly at telopeptide region have been known to involve in cross-linkage of collagen (Knott and Bailey, 1998). Partial elimination of telopeptides by pepsin more likely resulted in some difference in amino acid composition between ASC and PSC (Matmaroh et al., 2011).

\section{Protein patterns}

Both ASC and PSC from scales of golden carp had $\alpha$-and $\beta$-chains as the major constituents (Fig. 1). The $\alpha_{1}$ - and $\alpha_{2}$-chains of ASC possessed MW of 117 and $108 \mathrm{kDa}$, respectively. For PSC, $\alpha_{1}$ - and $\alpha_{2}$-chains showed slightly lower MW (114 and $106 \mathrm{kDa}$, respectively), compared to those of ASC. Similar results were reported by Nalinanon et al. (2010), in which $\alpha_{1}$ and $\alpha_{2}$ chains of PSC showed marginally lower MW than those of ASC from arabesque greenling skin. With pepsin treatment, shortening of the chain was therefore predicted due to the cleavage of telopeptide. It was noted that ratio of $\alpha_{1} / \alpha_{2}$ chain was 2:1, suggesting that both ASC and PSC belonged to type 1 . ASC from scales of some fish species including seabass (Chuaychan et al., 2015) and snakehead fish (Liu et al., 2008) was classified to be type I collagen. Furthermore, $\beta$-chain (dimer) and $\gamma$-chain (trimer) were also observed in both ASC and PSC, suggesting the presence of high MW crosslinks. Similar band intensity of $\gamma$-chain in both ASC and PSC were observed, but the band intensity of $\beta$-chain and $\alpha$-chain of PSC was more intense than that found in ASC (Nalinanon et al., 2007). This could suggest that pepsin was able to cleave the telopeptide region, thereby releasing more $\alpha$-and $\beta$-chains in PSC. Moreover, there were no low MW proteins in both collagens. Thus, no degradation took place and there was negligible contamination of non-collagenous proteins in both collagens.

\section{Fourier-transform infrared spectra}

FTIR spectra of ASC and PSC depicted in Fig. 2A confirmed the distinctive peaks for amide A, B, I, II and III. In general, amide bands I, II, and III are responsible 


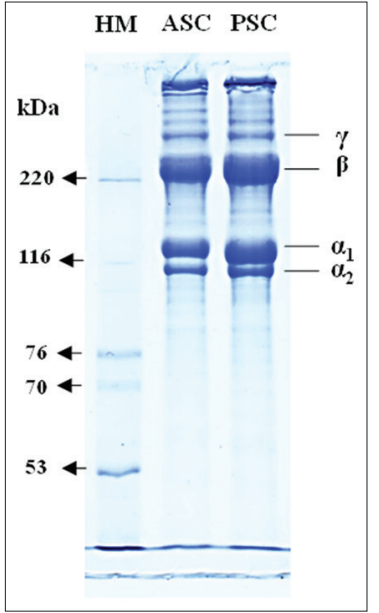

Fig 1. SDS-PAGE patterns of acid soluble collagen (ASC) and pepsin soluble collagen (PSC) from scales of golden carp. HM: high molecular weight marker.

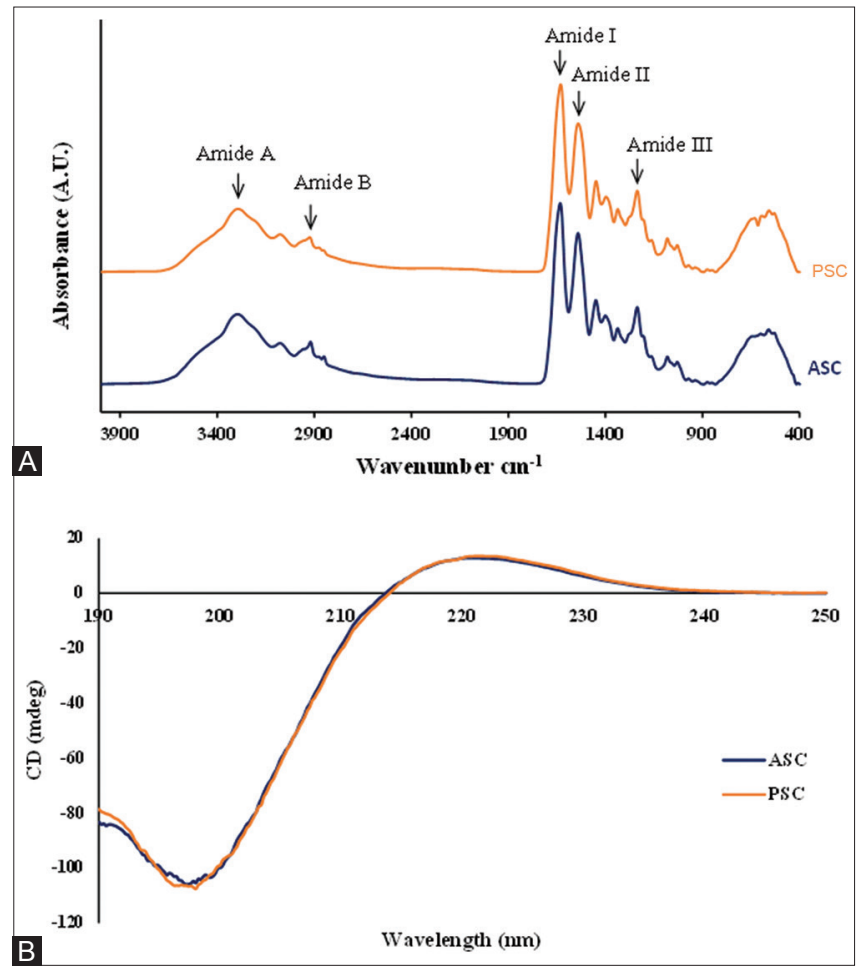

Fig 2. Fourier transform infrared spectra $(A)$ and $C D$ spectra $(B)$ of acid soluble collagen (ASC) and pepsin soluble collagen (PSC) from scales of golden carp.

for molecule order and formation of triple helical structure in collagen. Those are related with the stretching of $\mathrm{C}=\mathrm{O}$, bending of $\mathrm{N}-\mathrm{H}$ and stretching of $\mathrm{C}-\mathrm{N}$ interactions, respectively (Chen et al., 2016).

Amide I distinctive wavenumbers range from 1600 to $1700 \mathrm{~cm}^{-1}$ (Payne and Veis, 1988). ASC and PSC exhibited absorption peaks at 1637 and $1631 \mathrm{~cm}^{-1}$, respectively. The hydrogen bond between $\mathrm{N}-\mathrm{H}$ stretch (X position) and $\mathrm{C}=\mathrm{O}$ (Gly) of the fourth residue is accountable for the formation of triple helix (Zanaboni et al., 2000). The higher wavenumber observed in ASC suggested that the removal of telopeptide could lower the $\mathrm{C}=\mathrm{O}$ of PSC. Amide II-bond is due to the $\mathrm{C}-\mathrm{N}$ stretching vibration in combination with the $\mathrm{N}-\mathrm{H}$ in-plane bending (Barth and Zscherp, 2002). Amide II wavenumber of ASC and PSC were at 1548 and $1540 \mathrm{~cm}^{-1}$, respectively. PSC presented lower wavenumber of amide II than ASC. This suggested that there was more proportion of hydrogen bonds present in PSC due to the removal of amorphous telopeptide. This result confirms that PSC had increased molecular integrity, and this might be attributable to the elimination of a non-helical portion of telopeptide region by pepsin. Furthermore, amide III is related with $\mathrm{N}-\mathrm{H}$ in-plane bending and $\mathrm{C}-\mathrm{N}$ stretching of amide linkages. ASC and PSC exhibited similar wavenumbers (1237 and $1236 \mathrm{~cm}^{-1}$ ) of Amide III bands. The absorption band pattern of amide III was from wagging vibrations of $\mathrm{CH}_{2}$ groups from the proline side-chains and glycine backbone (Plepis et al., 1996).

Amide A bands associated with free $\mathrm{N}-\mathrm{H}$ stretching vibration occur in the range of $3400-3440 \mathrm{~cm}^{-1}$. ASC and PSC demonstrated wavenumbers of 3295 and $3292 \mathrm{~cm}^{-1}$, respectively. The shift in wavenumber to lower frequency was observed due to the involvement of $\mathrm{NH}$ group in hydrogen bonding (Fontaine-Vive et al., 2009). The results suggested that $\mathrm{NH}$ underwent $\mathrm{H}$-bond of helix in both ASC and PSC. The amide B bands that are responsible for asymmetrical stretching of $\mathrm{CH}_{2}$ (Abe and Krimm, 1972) were found at 2920 and $2924 \mathrm{~cm}^{-1}$ for ASC and PSC, respectively. ASC and PSC also showed band around $1451-1450 \mathrm{~cm}^{-1}$. The vibration of proline and hydroxyproline pyrrolidine ring attributed to the peak (Matmaroh et al., 2011). Peak ratio between amide III and $1454 \mathrm{~cm}^{-1}$ band was 0.98 and 0.99 for both ASC and PSC, respectively. Presence of triple-helical structure was indicated by the peak ratio of 1.0 (Benjakul et al., 2010). The results showed that both collagens were most likely in the native triple helical structure.

\section{CD spectroscopy}

The spectra of ASC and PSC were scanned in the range of 190-250 nm as shown in Fig. 2B. Rotatory maxima of ASC and PSC were at 220.8 and $221 \mathrm{~nm}$, respectively. Both the samples showed a negative peak at $198 \mathrm{~nm}$. Overall, ASC and PSC exhibited similar spectra having positive and negative peaks at 221 and $198 \mathrm{~nm}$, representing a characteristic feature of triple helical conformation (Tifany and Krimm, 1972). CD spectra represent backbone configuration of protein through absorption regions of peptide linkage. A minor difference was observed in ellipticity between ASC and PSC, suggesting some difference in the secondary conformation of both collagens, more likely due to telopeptide removal in PSC 
(Ogawa et al., 2004). Moreover, the denatured collagen possessed more distorted spectrum, reflected by the disappearance of positive peak at $221 \mathrm{~nm}$. Additionally, negative peak shifts to the lower absorption than $198 \mathrm{~nm}$ (Tifany and Krimm, 1972). The results confirmed that ASC and PSC had high structural integrity without denaturation.

\section{Zeta potential}

Zeta potential of both ASC and PSC from scales of golden carp at $\mathrm{pHs}$ ranging from 2 to 12 is shown in Fig. 3. With a $\mathrm{pH}$ range of $2-6$, both the collagen samples were positively charged, and the charge decreased continuously with increasing pHs. ASC and PSC had surface net charge of zero at $\mathrm{pH} 6.04$ and 6.22, respectively, which were estimated to be their isoelectric points (pI). Carboxylic group primarily from acidic amino acids such as glutamic acid and aspartic acid were found at higher extent (Table 1). At $\mathrm{pHs}$ above the $\mathrm{pI}$, both the samples had the shift in electric charge from positive to negative, and there was a further increase in negative charge as $\mathrm{pH}$ increased. The change in electric charge was governed by protonation and deprotonation of amino acids at different $\mathrm{pHs}$ as reported by Benjakul et al. (2010). Collagens from various sources showed different pIs. ASC from seabass skin had higher pI (6.64) (Sinthusamran et al., 2013), while ASC from skins of striped catfish had pI at 4.72 (Singh et al., 2011). This difference in $\mathrm{pI}$ might be due to the difference in distribution and composition of amino acid residues, predominantly on the surface domains of the collagen molecule.

\section{Thermal transition}

Thermal transition curves of ASC and PSC from golden carp scales are presented in Fig. 4. ASC and PSC had $\mathrm{T}_{\max }$ values of 37.67 and $37.83{ }^{\circ} \mathrm{C}$, respectively. $\mathrm{T}_{\max }$ values of ASC and PSC suggested that both have comparable secondary structure. Moreover, thermal stability of collagen was mainly governed by pyrrlidine rings and hydrogen bonds of hydroxyl group of hydroxyproline (Benjakul et al., 2010). It was noted that PSC had slightly higher $\Delta H$ values, compared to ASC. This might be due to the elimination of non-helical telopeptide region, resulting in the formation of more compact structure of PSC.

Consequently, PSC needed greater energy for destabilisation than ASC. The results were also influenced by the higher content of imino acids, especially hydroxyproline content in PSC (Table 1). ASC and PSC demonstrated lower $\mathrm{T}_{\max }$ values than type I collagens from scales of spotted golden goatfish $\left(41.58{ }^{\circ} \mathrm{C}\right)$ (Matmaroh et al., 2011) and seabass scales $\left(39.32^{\circ} \mathrm{C}\right)$ (Chuaychan et al., 2015). Nonetheless, ASC and PSC from scales of golden carp had higher $\mathrm{T}_{\max }$ values than ASC and PSC from silver carp scales $\left(29^{\circ} \mathrm{C}\right)$ (Zhang et al., 2010), ASC from scale of rohu and catla

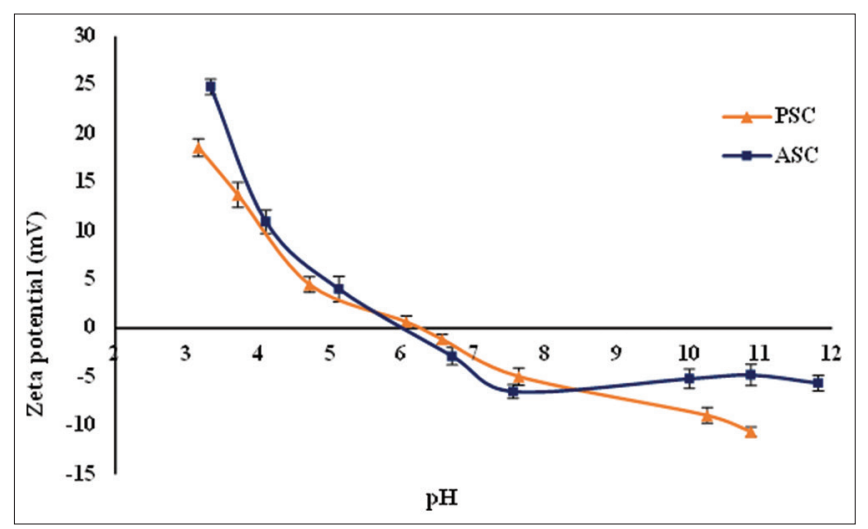

Fig 3. Zeta potential of acid soluble collagen (ASC) and pepsin soluble collagen (PSC) from scales of spotted golden carp at different pHs. Bars represent the standard deviation $(n=3)$.

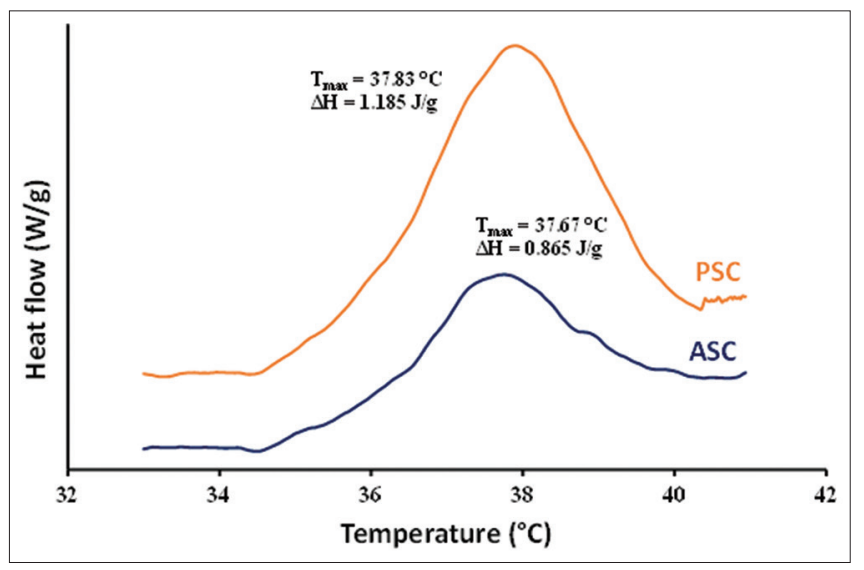

Fig 4. DSC thermogram of acid soluble collagen (ASC) and pepsin soluble collagen (PSC) from scales of golden carp.

$\left(36.5^{\circ} \mathrm{C}\right)$ (Pati et al., 2010) and PSC from the scale of bighead carp $\left(35.2^{\circ} \mathrm{C}\right)$ (Liu et al., 2012). Generally, the collagens from cold water dwelling fish contains lower amounts of hydroxyproline than those living in warm water (Muyonga et al., 2004). Lower thermal stability is obtained in the former. Denaturation temperatures of collagen is governed by fish species, age, seasons, habitat temperature, etc. (Duan et al., 2009; Muyonga et al., 2004).

\section{Solubility test}

\section{Effect of $\mathrm{pH}$}

The relative solubility of both ASC and PSC of golden carp scales at varying $\mathrm{pHs}$ is presented in Fig. 5A. pH had the marked effect on the relative solubility of both collagens. Both samples showed similar solubility pattern. Overall, both collagens were highly soluble in the $\mathrm{pH}$ range of 1-3. Likewise, similar solubility profile was reported for ASC and PSC from scales of seabass and snakehead (Chuaychan et al., 2015; Liu et al., 2012). At pH higher than 3 , solubility was drastically decreased and very low solubility was observed at $\mathrm{pH} 5$ and 6 . This decrease in solubility could be related to the increase in hydrophobic interaction 


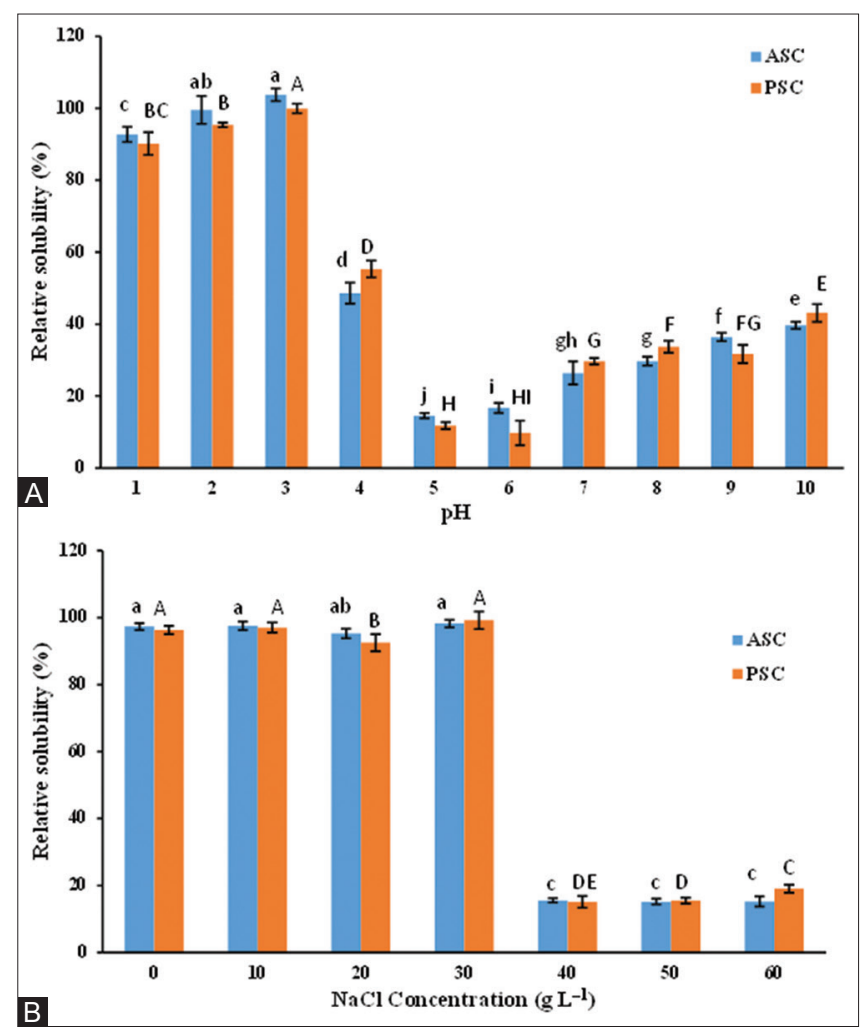

Fig 5. Relative solubility of acid soluble collagen (ASC) and pepsin soluble collagen (PSC) from scales of golden carp at different $\mathrm{pHs}$ (A) and $\mathrm{NaCl}$ concentrations, (B). Different lowercase or uppercase letters on the bars within the same sample indicate significant difference $(p<0.05)$.

between collagen molecules in a solution as described by Sinthusamran et al. (2013). Furthermore, there was a slight increase in solubility at alkaline $\mathrm{pH}$. The repulsive force of collagen molecules mediated by the negatively charged domains at $\mathrm{pH}$ above $\mathrm{pI}$ of both collagens contributed to the slight increase in solubility. Different solubility between both collagens was probably determined by the dissimilarity in amino acid composition, molecular properties, chain length and conformation (Kittiphattanabawon et al., 2005).

\section{Effect of $\mathrm{NaCl}$}

Relative solubility of ASC and PSC as influenced by different $\mathrm{NaCl}$ concentrations is shown in Fig. 5B. Both collagens remained soluble as $\mathrm{NaCl}$ was present up to $30 \mathrm{~g}$ $\mathrm{L}^{-1}(\mathrm{p}<0.05)$. No difference in solubility between ASC and PSC was noticable $(p>0.05)$. At a lower concentration of $\mathrm{NaCl}$, salt ions have weak interactions with the charge groups on proteins surface (Damodaran, 1996). Thus, low salt concentration did not have the effect on charge as well as solubility of collagen. Conversely, $\mathrm{NaCl}$ at high concentrations led to the drastic decline in solubility of ASC and PSC. The marked decreased in solubility might be caused by 'salting out' effect of collagen (Bae et al., 2008). At high salt concentration, hydrophobic interaction between proteins chains was enhanced. Simultaneously, the water was bound with salt to a higher extent. This resulted in precipitation of collagen (Damodaran, 1996).

\section{CONCLUSIONS}

ASC and PSC extracted from golden carp scales were characterised to be type I collagen. The aid of pepsin helped in increasing the yield of collagen up to 3.8 folds. Based on FTIR, CD and SDS-PAGE analyses, both collagens were in triple helical structure. There was a slight difference in amino acid composition as well as enthalpy for thermal transition between ASC and PSC, mainly mediated by the elimination of telopeptide by pepsin. Thus, collagen from scales of golden carp could serve as an alternative to those from mammalian sources. As a consequence, limitation of the latter associated with religious constraint and safety can be conquered.

\section{ACKNOWLEDGEMENT}

This work was supported by the Higher Education Research Promotion and National Research University Project of Thailand, Office of the Higher Education Commission and the Grant-in-Aid for dissertation from Graduate School, Prince of Songkla University, Thailand. The TRF Distinguished Research Professor grant were acknowledged for financial support.

\section{Author contributions}

A. M. M. A. performed experiments and analyses. SB formulated the hypothesis and designed the studies. H. K. contributed to the literature review and analysis of amino acid. All authors have approved the final version of the manuscript.

\section{REFERENCES}

Abe, Y., and S. Krimm. 1972. Normal vibrations of crystalline polyglycine I. Biopolymers. 11: 1817-1839.

AOAC. 2000. Official Methods of Analysis. $17^{\text {th }}$ ed. Association of Official Ananlytical Chemists, Arlington, VA.

Bae, I., K. Osatomi, A. Yoshida, K. Osako, A. Yamaguchi and K. Hara. 2008. Biochemical properties of acid-soluble collagens extracted from the skins of underutilised fishes. Food Chem. 108: 49-54.

Barth, A. and C. Zscherp. 2002. What vibrations tell about proteins. Q. Rev. Biophys. 35: 369-430.

Benjakul, S., Y. Thiansilakul, W. Visessanguan, S. Roytrakul, H. Kishimura, T. Prodpran and J. Meesane. 2010. Extraction and characterisation of pepsin-solubilised collagens from the skin of bigeye snapper (Priacanthus tayenus and Priacanthus macracanthus). J. Sci. Food Agric. 90: 132-138.

Bergman, I. and R. Loxley. 1963. Two improved and simplified methods for the spectrophotometric determination of hydroxyproline. Anal. Chem. 35: 1961-1965.

Chen, J., L. Li, R. Yi, N. Xu, R. Gao and B. Hong. 2016. Extraction 
and characterization of acid-soluble collagen from scales and skin of tilapia (Oreochromis niloticus). LWT Food Sci. Technol. 66: 453-459.

Chuaychan, S., S. Benjakul and H. Kishimura. 2015. Characteristics of acid-and pepsin-soluble collagens from scale of seabass (Lates calcarifer). LWT Food Sci. Technol. 63: 71-76.

Damodaran, S. 1996. Amino acids, peptides, and proteins. In: O. R. Fennema (Ed.), Food Chemistry, Marcel Dekker, Inc., New York, pp. 321-429.

Duan, R., J. Zhang, X. Du, X. Yao and K. Konno. 2009. Properties of collagen from skin, scale and bone of carp (Cyprinus carpio). Food Chem. 112: 702-706.

FAO. 2016. Fishery and Aquaculture Statistics 2014, Food and Agricultural Organization of the United Nation, Rome, pp. 189-194.

Fontaine-Vive, F., F. Merzel, M. R. Johnson and G. J. Kearley. 2009. Collagen and component polypeptides: Low frequency and amide vibrations. Chem. Phys. 355: 141-148.

Gornall, A. G., Bardawill, C. J. and David, M. M. 1949. Determination of serum proteins by means of the biuret reaction. J. Biol. Chem. 177: 751-766.

Jongjareonrak, A., S. Benjakul, W. Visessanguan, T. Nagai and M. Tanaka. 2005. Isolation and characterisation of acid and pepsin-solubilised collagens from the skin of brownstripe red snapper (Lutjanus vitta). Food Chem. 93: 475-484.

Kielty, C. M., I. Hoplinson and M. E. Grant. 1993. In: Royce, P. M. and B. Steinmann (Eds.), Part I: Connective Tissue and Its Heritable Disorders, Wiley-Interscience, New York, pp. 103-147.

Kittiphattanabawon, P., S. Benjakul, W. Visessanguan, T. Nagai and M. Tanaka. 2005. Characterisation of acid-soluble collagen from skin and bone of bigeye snapper (Priacanthus tayenus). Food Chem. 89: 363-372.

Knott, L. and A. J. Bailey. 1998. Collagen cross-links in mineralizing tissues: A review of their chemistry, function, and clinical relevance. Bone. 22: 181-187.

Laemmli, U. K. 1970. Cleavage of structural proteins during the assembly of the head of bacteriophage T4. Nature. 227: 680-685.

Li, C., Z. Zhong, Q. Wan, H. Zhao, H. Gu and S. Xiong. 2008. Preparation and thermal stability of collagen from scales of grass carp (Ctenopharyngodon idellus). Eur. Food Res. Technol. 227: 1467-1473.

Liu, D., L. Liang, J. M. Regenstein and P. Zhou. 2012. Extraction and characterisation of pepsin-solubilised collagen from fins, scales, skins, bones and swim bladders of bighead carp (Hypophthalmichthys nobilis). Food Chem. 133: 1441-1448.

Liu, W. T., Y. Zhang, G. Y. Li, Y. Q. Miao and X. H. Wu. 2008. Structure and composition of teleost scales from snakehead Channa argus (Cantor) (Perciformes: Channidae). J. Fish Biol. 72: 1055-1067.

Lowry, O. H., N. J. Rosebrough, A. L. Farr and R. J. Randall. 1951. Protein measurement with the Folin phenol reagent. J. Biol. Chem. 193: 265-275.

Matmaroh, K., S. Benjakul, T. Prodpran, A. B. Encarnacion and H. Kishimura. 2011. Characteristics of acid soluble collagen and pepsin soluble collagen from scale of spotted golden goatfish
(Parupeneus heptacanthus). Food Chem. 129: 1179-1186.

Muyonga, J.H., C. G. B. Cole and K. G. Duodu. 2004. Characterisation of acid soluble collagen from skins of young and adult nile perch (Lates niloticus). Food Chem. 85: 81-89.

Nagarajan, M., S. Benjakul, T. Prodpran, P. Songtipya and H. Kishimura. 2012. Characteristics and functional properties of gelatin from splendid squid (Loligo formosana) skin as affected by extraction temperatures. Food Hydrocoll. 29: 389-397.

Nalinanon, S., S. Benjakul and H. Kishimura. 2010. Collagens from the skin of arabesque greenling (Pleurogrammus azonus) solubilized with the aid of acetic acid and pepsin from albacore tuna (Thunnus alalunga) stomach. J. Sci. Food Agric. 90: 1492-1500.

Nalinanon, S., S. Benjakul, W. Visessanguan and H. Kishimura. 2007. Use of pepsin for collagen extraction from the skin of bigeye snapper (Priacanthus tayenus). Food Chem. 104: 593-601.

Ogawa, M., R. J. Portier, M. W. Moody, J. Bell, M. A. Schexnayder and J. N. Losso. 2004. Biochemical properties of bone and scale collagens isolated from the subtropical fish black drum (Pogonia cromis) and sheepshead seabream (Archosargus probatocephalus). Food Chem. 88: 495-501.

Pati, F., B. Adhikari and S. Dhara. 2010. Isolation and characterization of fish scale collagen of higher thermal stability. Bioresour. Technol. 101: 3737-3742.

Payne, K. J. and A. Veis. 1988. Fourier transform ir spectroscopy of collagen and gelatin solutions: Deconvolution of the amide I band for conformational studies. Biopolymers. 27: 1749-1760.

Plepis, A. M. D., G. Goissis and D. K. Das-Gupta. 1996. Dielectric and pyroelectric characterization of anionic and native collagen. Polym. Eng. Sci. 36: 2932-2938.

Rizk, M. A. and N. Y. Mostafa. 2016. Extraction and characterization of collagen from buffalo skin for biomedical applications. Orient. J. Chem. 32: 1601-1609.

Singh, P., S. Benjakul, S. Maqsood and H. Kishimura. 2011. Isolation and characterisation of collagen extracted from the skin of striped catfish (Pangasianodon hypophthalmus). Food Chem. 124: $97-105$.

Sinthusamran, S., S. Benjakul and H. Kishimura. 2013. Comparative study on molecular characteristics of acid soluble collagens from skin and swim bladder of seabass (Lates calcarifer). Food Chem. 138: 2435-2441.

Tifany, M. L. and S. Krimm. 1972. Effect of temperature on the circular dichroism spectra of polypeptides in the extended state. Biopolymers. 11: 2309-2316.

Zall, R. R. (Ed.). 2004. Unconventional techniques to deal with waste recovery or treatment schemes. Managing Food Industry Waste. Blackwell Publishing, lowa, pp. 105-113.

Zanaboni, G., A. Rossi, A. M. T. Onana and R. Tenni. 2000. Stability and networks of hydrogen bonds of the collagen triple helical structure: Influence of $\mathrm{pH}$ and chaotropic nature of three anions. Matrix Biol. 19: 511-520.

Zhang, J., R. Duan, C. Ye and K. Konno. 2010. Isolation and characterization of collagens from scale of silver carp (Hypophthalmichthys Molitrix). J. Food Biochem. 34: 1343-1354. 